\title{
Rotation Invariant Texture Classification Using Circular Gabor Filter Banks
}

\author{
Qingbo Yin ${ }^{1,2}$ and Jong Nam Kim ${ }^{1}$ \\ ${ }^{1}$ Division of Electronic Computer and Telecommunication Engineering, Pukyong National \\ University, 599-1 Daeyeon-dong Nam-gu, Busan, 608-737, Korea \\ Jongnan@pknu.ac.kr \\ ${ }^{2}$ College of Computer Science and Technology, Harbin Engineering University, Harbin, \\ 150001, P.R. China \\ yinqingbo@hrbeu.edu.cn
}

\begin{abstract}
This paper presents a new method for rotation invariant texture classification based on the circular Gabor wavelets. A circular Gabor filter bank is proposed to decompose an image into multiple scales and be rotation invariant. By the mean and variance of the circular Gabor filtered image, a discriminant can be found to classify rotated images. In the primary experiments, comparatively high correct classification rates were obtained using a large test sample set.
\end{abstract}

\section{Introduction}

Texture analysis is a fundamental issue in image analysis and computer vision. The Gabor function can be appropriately considered as an orientation and scale tunable detector. The banks of Gabor filters are a group of wavelets, which one can capture the signal or feature at a specific frequency and a specific orientation. There are several approaches based on Gabor filters, which focus on the rotation invariant texture in [1-3]. But, most of them only work well on a small database and need a lot of samples for training.

The motivation of this paper is to classify rotation-invariant textures in a large texture database. The proposed algorithm is based on circular Gabor filter responses, in which each texture has the corresponding global features. Then, a similarity measure is used to compare the unknown samples with the feature of known textures. The primary experiments have proven this approach performs well in applications.

\section{Circular Gabor Wavelet and Classification}

For the filter to be rotation invariant, it is necessary that the sinusoidal grating varies in all orientations. In the circular Gabor filter, both Gaussian and grating vary only in a radial direction from origin, such that the filter is completely circularly symmetric. The circular Gabor filter is defined as[1]: 


$$
C g(x, y)=\frac{1}{2 \pi \sigma^{2}} \exp \left[-\frac{\left(x^{2}+y^{2}\right)}{2 \sigma^{2}}\right] \cdot \exp \left[j 2 \pi W\left(\sqrt{x^{2}+y^{2}}\right)\right]
$$

Where $W$ is the required centered frequency of the circular Gabor filter, and $\sigma=\sigma_{x}=\sigma_{y}$. The frequency domain representation of the circular Gabor filter is given by:

$$
C G(u, v)=\frac{\sqrt{2 \pi}}{2} \alpha \cdot \exp \left[-\frac{\left(\sqrt{u^{2}+v^{2}}-W\right)^{2}}{2 \alpha^{2}}\right], \alpha=\frac{1}{2 \pi \sigma}
$$

\subsection{Circular Gabor Wavelet and Parameter Selection}

A class of self-similar functions like the wavelet is considered as the circular Gabor filter bank or circular Gabor wavelet in the following discussion. Let $\operatorname{Cg}(x, y)$ be the mother Gabor wavelet, then this self-similar filter dictionary can be obtained by appropriate dilations and rotations of $C g(x, y)$ through the generating function:

$$
\begin{gathered}
C g_{m}(x, y)=a^{-m} \operatorname{Cg}\left(x^{\prime}, y^{\prime}\right), a>1, m=1, \ldots, S \\
x^{\prime}=a^{-m} x, \text { and } y^{\prime}=a^{-m} y
\end{gathered}
$$

Where $a$ is a scale factor, and the factor $a^{-m}$ is meant to ensure that the energy is independent of $m . S$ is the number of scales in the multiresolution decomposition.

Let $W_{l}$ and $W_{h}$ denote the lower and upper center frequencies of interest. Then, the design strategy is to ensure that the half peak magnitude cross-sections of the filter responses in the frequency spectrum touch each other. This results in the following formulas for computing the filter parameters $\sigma$ and $W$ :

$$
a=\left(W_{h} / W_{l}\right)^{1 / s-1}, \text { or } W_{h}=W_{l} \cdot a^{(s-1)}, \sigma=(3 \sqrt{2 \ln 2}) /\left(2 \pi W_{h}\right)
$$

\subsection{Texture Representation and Rotation Invariant Measurement}

Given an image $I(x, y)$ of size $M * N$, its discrete circular Gabor transform is given by convolution:

$$
H_{m}(x, y)=\sum_{p} \sum_{q} I(x-p, y-q) C g_{m}^{*}(p, q), m=1, \ldots, S
$$

Where $p, q$ is the filter mask size, $C g_{m}^{*}(p, q)$ is the complex conjugate of $C g_{m}$. 
It is assumed that the texture regions are spatially homogeneous. The mean $\mu_{m}$ and standard deviation $\sigma_{m}$ of the magnitude of the transform coefficients are used to construct global features to represent the homogeneous textures.

$$
\mu_{m}=\frac{\sum_{M} \sum_{N}\left|H_{m n}(x, y)\right|}{M * N}, \sigma_{m}=\frac{\sqrt{\sum_{x} \sum_{y}\left(\left|H_{m}(x, y)\right|-\mu_{m}\right)^{2}}}{M * N}, m=1, \ldots, S
$$

Now, the direct distance metric can be defined between the query image $Q$ and a target image $T$ in the database as:

$$
d_{m}^{\mu}(Q, T)=\left|\mu_{m}^{Q}-\mu_{m}^{T}\right|, d_{m}^{\sigma}(Q, T)=\left|\sigma_{m}^{Q}-\sigma_{m}^{T}\right|
$$

Three differential factors can be defined as:

$$
d f_{\mu}=\frac{\sum_{m=1}^{S}\left(d_{m}^{\mu}\right)^{2}}{\sum_{m=1}^{S}\left(\mu_{m}^{T}\right)^{2}}, d f_{\sigma}=\frac{\sum_{m=1}^{S}\left(d_{m}^{\sigma}\right)^{2}}{\sum_{m=1}^{S}\left(\sigma_{m}^{T}\right)^{2}}, d f_{D}=\sum_{m=1}^{S}\left(d_{m}^{\mu}\right)^{2}+\sum_{m=1}^{S}\left(d_{m}^{\sigma}\right)^{2}
$$

Actually, the texture similarity measurement is accomplished by defining the differential measurement:

$$
d f=\min \left(d f_{\sigma} * d f_{\mu} * d f_{D}\right)
$$

This similarity measurement is rotation invariant, and is smallest if $Q$ is the same as or very similar to $T$.

\section{Experiments}

The experiments are carried out on a texture database from the Brodatz texture album, which is comprised of 111 texture images. Each center portion of size $256 \times 256$ from each respective texture image is used in the training phase. Texture classification is done with a total of 1998 rotated texture images in steps of $10^{\circ}$ up to $180^{\circ}$ for various scale values of Gabor decomposition (scale is from 2 up to 10). The center frequencies for Gabor filters are 0.02-0.48 and are separated by one octave. In Table 1, the results of comparisons with other main methods [2-3] are given. Other schemes need a large training for each class of images. However, the proposed approach only needs a sample of each class for training at 0 angle, then can work well. Nevertheless, the proposed approach can deal with 111 classes with a correct classification rate of more than $97 \%$, only using scale $=7$ and 14 features. 
Table 1. The performance comparisons between the proposed approach and other schemes

\begin{tabular}{|c|c|c|c|c|c|}
\hline \multirow[b]{2}{*}{ Method } & \multicolumn{3}{|c|}{ Samples } & \multirow[b]{2}{*}{$\begin{array}{c}\text { The } \\
\text { number of } \\
\text { features }\end{array}$} & \multirow[b]{2}{*}{$\begin{array}{c}\text { Correct } \\
\text { classification } \\
\text { rate }\end{array}$} \\
\hline & $\begin{array}{c}\text { Number of classes } \\
\text { /total of images }\end{array}$ & $\begin{array}{l}\text { Number of } \\
\text { each class } \\
\text { for training }\end{array}$ & $\begin{array}{l}\text { Number of } \\
\text { each class } \\
\text { for testing }\end{array}$ & & \\
\hline $\begin{array}{c}\text { Space- } \\
\text { frequency } \\
\text { model [3] }\end{array}$ & $\begin{array}{c}109 / \\
(872 * 2=1744)\end{array}$ & 8 & 8 & - & $80.4 \%$ \\
\hline $\begin{array}{c}\text { Arivazhagan's } \\
\text { method [2] }\end{array}$ & $\begin{array}{c}112 / \\
(112 * 36=4032)\end{array}$ & 1 & 36 & 100 & $93.7 \%$ \\
\hline $\begin{array}{l}\text { Proposed } \\
\text { approach }\end{array}$ & $\begin{array}{c}111 / \\
(111 * 18+111=210 \\
9)\end{array}$ & 1 & 18 & 14 & $97.7 \%$ \\
\hline
\end{tabular}

\section{Conclusions}

A rotation invariant texture classification scheme based on the circular Gabor filter is developed for a reasonably large (111 classes) texture databases. By the mean and variance of the Gabor filtered image, a discriminant can be found to classify rotated images. The primary experiments have proven that the proposed approach is effective for rotation invariant texture classification.

\section{Acknowledgements}

This work was supported by The Regional Research Centers Program (Research Center for Logistics Information Technology), granted by the Korean Ministry of Education \& Human Resources Development.

\section{References}

1. Porter, R., Canagarajah, N.: Robust rotation-invariant texture classification: Wavelet, Gabor filter and GMRF based schemes. IEE Proceedings of Image Signal Processing 144 (3), 1997.

2. Arivazhagan, S., Ganesan L., and Padam Priyal, S.: Texture classification using Gabor wavelets based rotation invariant features. Pattern Recognition Letters, Vol.27(16), (2006) 1976-1982

3. Haley, G.M., Manjunath, B.S.: Rotation-invariant texture classification using a complete space-frequency model. IEEE Transactions on Image Processing 8 (2), (1999) 169-255. 